OPEN

SUBJECT AREAS:

MAGNETIC DEVICES

BIOSENSORS

BIONANOELECTRONICS

DIAGNOSTIC MARKERS

Received

18 February 2013

Accepted

1 July 2013

Published

19 July 2013

Correspondence and requests for materials should be addressed to

A.J.W. (ajwyrobek@

lbl.gov) or S.X.W.

(sxwang@stanford.

edu)

\title{
Nanosensor dosimetry of mouse blood proteins after exposure to ionizing radiation
}

Dokyoon Kim' ', Francesco Marchetti ${ }^{8,9}$, Zuxiong Chen', Sasa Zaric', Robert J. Wilson' , Drew A. Hall', Richard S. Gaster ${ }^{3}$, Jung-Rok Lee ${ }^{4}$, Junyi Wang ${ }^{2}$, Sebastian J. Osterfeld ${ }^{5}$, Heng Yu ${ }^{5}$, Robert M. White', William F. Blakely', Leif E. Peterson7, Sandhya Bhatnagar ${ }^{8}$, Brandon Mannion ${ }^{8}$, Serena Tseng ${ }^{8}$, Kristen Roth ${ }^{8}$, Matthew Coleman ${ }^{8}$, Antoine M. Snijders ${ }^{8}$, Andrew J. Wyrobek ${ }^{8} \&$ Shan X. Wang ${ }^{1,2}$

\footnotetext{
${ }^{1}$ Department of Materials Science and Engineering, ${ }^{2}$ Department of Electrical Engineering, ${ }^{3}$ Department of Bioengineering, ${ }^{4}$ Department of Mechanical Engineering, Stanford University, Stanford, California 94305, ${ }^{5}$ MagArray Inc., 1230 Bordeaux Drive, Sunnyvale, CA 94089, ${ }^{6}$ Armed Forces Radiobiology Research Institute, Uniformed Services University of the Health Sciences, Bethesda, Maryland 20889, ${ }^{7}$ Center for Biostatistics, The Methodist Hospital Research Institute, Houston, Texas $77030,{ }^{8}$ Life Sciences Division, Lawrence Berkeley National Laboratory, Berkeley, California 94720, USA, ${ }^{9}$ Environmental Health Science Research Bureau, Health Canada, Ottawa, ON K1A OK9, Canada.
}

Giant magnetoresistive (GMR) nanosensors provide a novel approach for measuring protein concentrations in blood for medical diagnosis. Using an in vivo mouse radiation model, we developed protocols for measuring Flt3 ligand (Flt3lg) and serum amyloid A1 (Saa1) in small amounts of blood collected during the first week after X-ray exposures of sham, $0.1,1,2,3$, or 6 Gy. Flt3lg concentrations showed excellent dose discrimination at $\geq 1$ Gy in the time window of 1 to 7 days after exposure except 1 Gy at day 7. Saal dose response was limited to the first two days after exposure. A multiplex assay with both proteins showed improved dose classification accuracy. Our magneto-nanosensor assay demonstrates the dose and time responses, low-dose sensitivity, small volume requirements, and rapid speed that have important advantages in radiation triage biodosimetry.

I xposure to ionizing radiation can, depending on dose and dose rate, induce immediate and persistent damage to internal organs including the lymphatic, haematopoietic, gastrointestinal, and central nervous systems $^{1,2}$. An accurate and rapid measurement of dose is crucial for informed medical response and management as well as effective medical triage ${ }^{3}$. Although personal dosimeters such as film badges or thermoluminescent dosimeters provide accurate radiation dosimetry in the facilities using a regulated radiation source, alternative methods are required to assess exposure dose in large-scale nuclear incidents and radiation accidents. Several biological methods have been proposed to assess an individual's absorbed dose retrospectively ${ }^{4-6}$. Frequency and duration of nausea, vomiting, and diarrhea have been proposed, but these physiological symptoms are not well correlated with the absorbed dose $e^{4}$. The dicentric chromosome assay remains the gold standard for assessing radiation dose. It measures chromosome aberrations in peripheral blood lymphocytes that are highly correlated with the absorbed dose ${ }^{5}$. However, cytogenetic methods are time-consuming, and thus are not well suited for incidents involving mass casualty where immediate triage decisions are required ${ }^{6}$. Alternative molecular approaches, such as the analyses of transcriptomic and proteomic changes (so-called molecular biomarkers) of radiation exposure, have been studied extensively due to their potential for high-throughput and rapid assays ${ }^{5,6}$.

A biomarker is a biomolecule whose concentration varies in a predictable manner with changes in biological state, with demonstrated utility to guide personalized medical treatment of patients ${ }^{7-9}$. Several detection methods and platforms including enzyme-linked immunosorbent assay (ELISA) ${ }^{10-12}$, nanomaterial-based sensors ${ }^{13-17}$, and electrochemical sensors ${ }^{18,19}$ have been developed to track the changes of biomarkers. However, those developed to date are generally limited in their performance due to insufficient sensitivity, poor scalability, lack of multiplex capability, and/or physiologically incompatible working conditions ${ }^{12,16,20}$. For example, ELISA, the most widely used immunoassay, makes use of colorimetric or fluorescent read-out that suffers from inherent optical absorption or autofluorescence of many biological species coexisting in samples ${ }^{20}$. Therefore, there remains a need for 


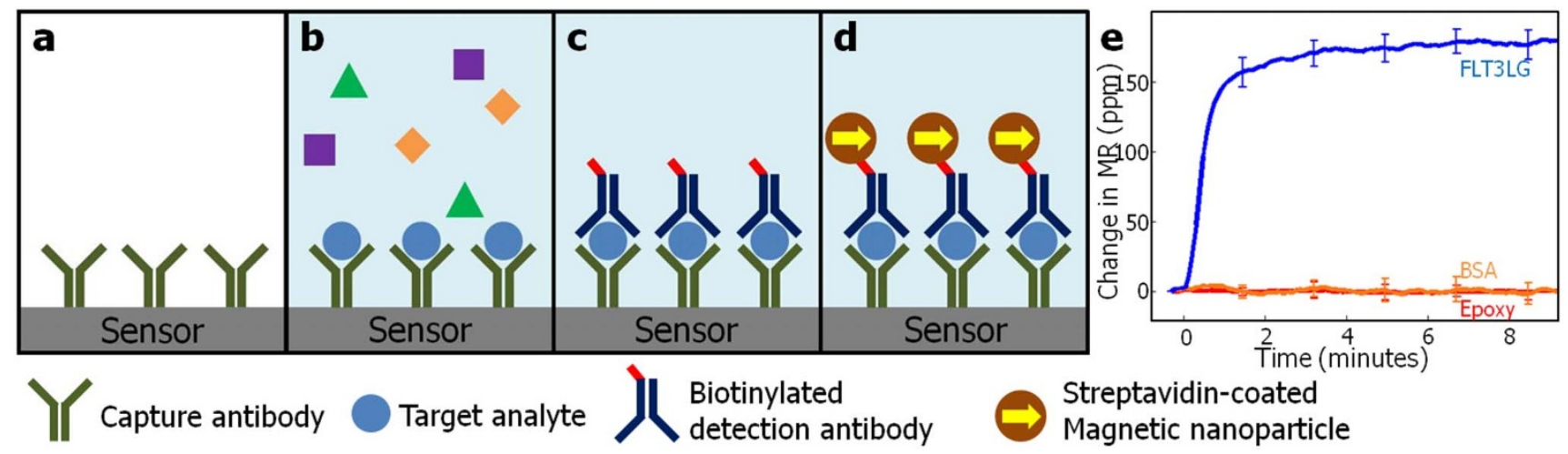

Figure $1 \mid$ (a-d) A schematic of magneto-nanosensor biochip immunoassay: (a) Capture antibodies are immobilized covalently on the sensor surface. (b) Target antigens are captured and noncomplementary antigens are subsequently washed away. (c) Addition of biotinylated detection antibody forms a sandwich structure. (d) Streptavidin-coated magnetic nanoparticles bound to the biotinylated detection antibody produce stray magnetic field. (e) An example of real-time binding curve showing the change in magnetoresistance (MR) in parts per million (ppm) over time for $500 \mathrm{pg} / \mathrm{ml} \mathrm{Flt3lg}$ (blue) compared with BSA negative control (orange) and epoxy reference (red). Error bars are \pm 1 standard deviation.

innovative ways to overcome the disadvantages of the current detection platforms for radiation biodosimetry in mass casualty exposures to ionizing radiation.

Blood is a preferred tissue for radiation biodosimetry because collection is minimally invasive and can be performed in non-clinical settings. Monitoring concentrations and changes in blood proteins is a well-accepted approach in medicine to guide personalized treatment. Over 200 proteins have been identified as radiation-responsive in tissues or cell culture after exposure to ionizing radiation, and our lab proposed a prioritized list of proteins as candidates for the assessment of radiation dose $\mathrm{e}^{21}$.

Major challenges in the use of protein biomarkers for radiation biodosimetry are the technical limitations in evaluating radiation responses of many blood proteins simultaneously with small blood volumes, sensitivity, technical ease, and short turn-around time. Our lab has developed magneto-nanosensors with multiplex capability for ultra-sensitive and matrix-independent detection of protein biomarkers using arrays of GMR spin-valves ${ }^{20,22-24}$. GMR is a quantum mechanical effect in which a change in the external magnetic field induces a change in the electrical resistance of a thin-film layered structure composed of at least two ferromagnetic layers. GMR spinvalve sensors are promising for handheld diagnostic devices due to their cost-effective microfabrication technology and existing manufacturing infrastructure in the hard disk drive industry ${ }^{25}$. Similar to ELISA, the magneto-nanosensor relies on a sandwich structure of the target analyte between the capture and detection antibodies. However, measurable signal, the resistance change of the spin-valve sensors, is generated by changes in the local magnetic fields from subsequently added superparamagnetic nanoparticles. The signal is in real-time, electrically processable, and more convenient to integrate with electronic read-out system than the optical signal from ELISA $^{20,22}$

The mouse is a well accepted mammalian model for identifying and validating biomarkers for radiation dosimetry after whole or partial body exposures to ionizing radiation ${ }^{26-28}$. For our current study, we developed a novel magneto-nanosensor immunoassay for Flt3lg and Saa1 in peripheral blood and tested it using the in vivo mouse radiation model. Flt3lg is an early-acting cytokine that regulates proliferation and differentiation of various haematopoietic cells, such as myeloid, lymphoid progenitor, and dendritic cells $\mathrm{s}^{29-32}$, and may be very useful in discerning the functional status of bone marrow $^{31,32}$. Saal is a member of serum amyloid A acute-phase protein family whose plasma level increases as much as a thousandfold with active inflammation ${ }^{33-35}$ and after exposure to ionizing radiation $^{36}$.
In this paper, we measured the dose and time dependent changes in Flt3lg and Saal concentrations in serial samplings of small amounts of blood $(\sim 25 \mu \mathrm{l})$ collected from live animals during the first week after exposure. Our magneto-nanosensor assay results are highly correlated with concurrent ELISA data, and our nanosensor platform responds linearly over wide concentration ranges. The nanosensor showed dose separation for each exposure group in the range of 1 to 6 Gy during the first week after exposure, and detected an increase of Flt3lg concentration after exposure to $1 \mathrm{~Gy}$, as evidence of its sensitivity at low doses. Our findings demonstrate the utility of our novel magneto-nanosensor platform for selecting candidate plasma proteins for radiation biodosimetry and for performing triage on large numbers of persons rapidly to identify individuals with radiation exposures that require immediate medical intervention.

\section{Results}

Detection scheme of magneto-nanosensor. The detection strategy for blood proteins (Fig. 1) relies on a sandwich approach that uses a well-characterized capture antibody immobilized onto a nanosensor surface $^{22}$. Blood proteins in solution that bind to the capture antibody are tagged with magnetic nanoparticles using biotin-streptavidin chemistry. When an external oscillating magnetic field is applied, stray fields induced from the bound magnetic nanoparticles induce resistance changes in the GMR spin-valve sensor located beneath the platform surface. The resistance changes are proportional to the local concentration of nanoparticles on the sensor surface, and are measured in real-time using a custom designed electric read-out system $^{37,38}$. A typical binding curve obtained by spiking Flt3lg into a dilution buffer at a concentration of $500 \mathrm{pg} / \mathrm{ml}$ and measuring signals from each sensor for about a 10 minute period is shown in Fig. 1e. Signals from Flt3lg capture antibody-coated sensors (blue) showed a magneto-resistance change of $\sim 180 \mathrm{ppm}$, while negative control sensors coated with BSA (orange) and reference sensors covered by epoxy (red) showed almost no signals. Additionally, biotin-coated sensors were used as positive functional control sensors and showed a signal around $\sim 1,100$ ppm (Supplementary Fig. S1).

Nanosensor calibration of radiosensitive blood proteins. We prepared standard curves to calibrate the nanosensor measurement of Flt3lg and Saal in mouse plasma samples (Fig. 2). The standard curves were generated from measurements where each biomarker was spiked in various concentrations into PBS. The standard dilutions of mouse plasma samples were chosen so that all mouse plasma data were within the linear range of the standard curve. The Flt3lg standard curve had a linear dynamic range of more than two orders of magnitude $(20 \sim 3,000 \mathrm{pg} / \mathrm{ml})$ with an $\mathrm{R}^{2}$ value of 0.98 
a

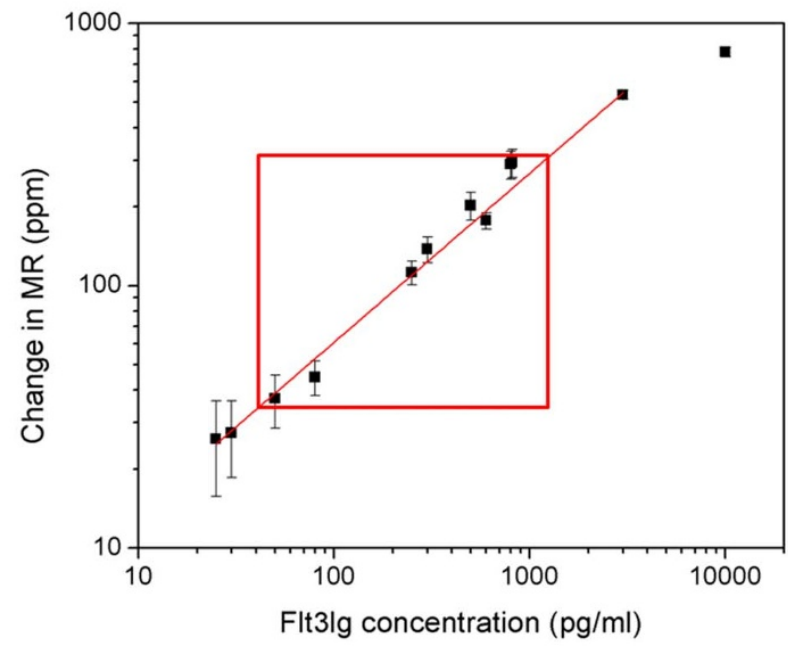

b

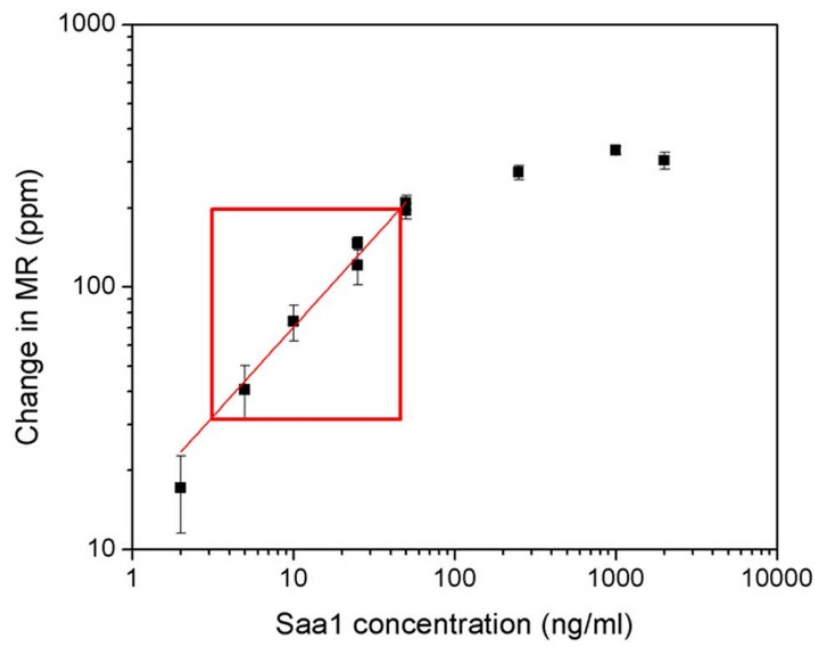

Figure $2 \mid$ Standard curves measured on magneto-nanosensor chips for mouse (a) Flt3lg and (b) Saal. Red boxes indicate the ranges of magnetonanosensor chip signal and biomarker concentration used in the assays performed in this work. Error bars are \pm 1 standard deviation.

(Fig. 2a). Our magneto-nanosensor assay has a detection limit similar to that of a commercially available ELISA kit with the same capture and detection antibodies (R\&D systems, DY427), but maintained a linear increase in signal even at concentrations higher than $1,000 \mathrm{pg} /$ $\mathrm{ml}$. On the other hand, the linear dynamic range of the Saal standard curve was from 3 to $50 \mathrm{ng} / \mathrm{ml}$ with an $\mathrm{R}^{2}$ value of 0.98 (Fig. 2b), and signals began to saturate at concentrations higher than $\sim 100 \mathrm{ng} / \mathrm{ml}$. The red boxes in Fig. 2 indicate the ranges of magneto-nanosensor chip signal we used for the Flt3lg and Saal assay.

Verification of magneto-nanosensor measurements using ELISA. The magneto-nanosensor immunoassay was significantly correlated with conventional ELISA measurements ( $\mathrm{p}<0.0001$ ). ELISA experiments were done using the same capture and detection antibodies used for the magneto-nanosensor immunoassay, although the capture antibody for ELISA was incubated overnight on a polystyrene plate rather than being covalently immobilized as it was on our nanosensor chip. Fig. 3 shows the scatter plots of each biomarker in spiked samples for both ELISA and magneto-nanosensor chip measurements. Spearman's rank correlation coefficient $(\rho)$ was calculated to

a

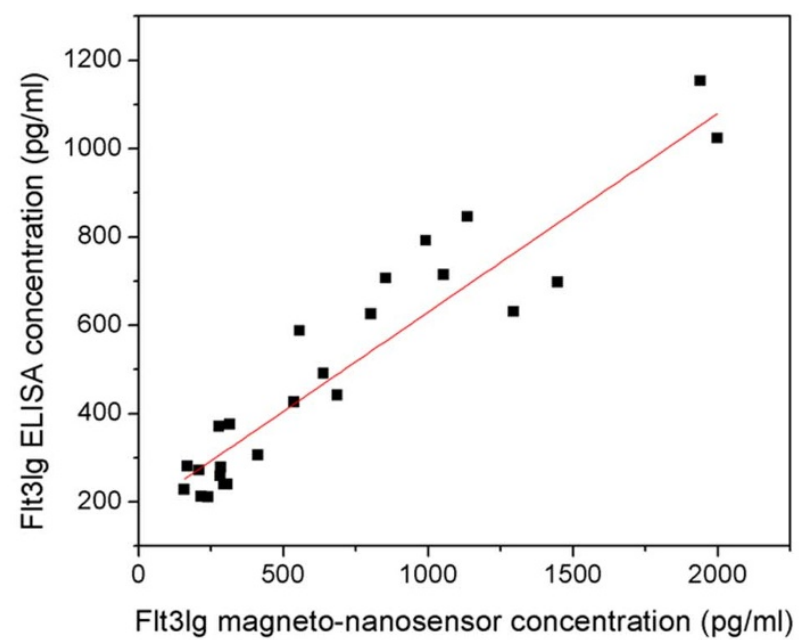

check the correlation between the ELISA and magneto-nanosensor measurements, and the calculated $\rho$ values were $0.915(\mathrm{p}<0.0001)$ for Flt3lg and $0.845(\mathrm{p}<0.0001)$ for Saa1, respectively.

Dose response in mouse plasma at day 1 after irradiation. Using the standard curves shown in Fig. 2, we tested the ability of our magneto-nanosensor platform to detect the effects of 2 and $6 \mathrm{~Gy}$ total body irradiation (TBI) on Flt3lg and Saal concentrations in peripheral blood plasma obtained from our in vivo mouse radiation model. We performed the immunoassay on a single magnetonanosensor biochip because we did not observe any cross-reaction between Flt3lg and Saa1. A total of 31 mouse plasma samples collected 24 hours after irradiation (sham, $\mathrm{n}=13 ; 2 \mathrm{~Gy}, \mathrm{n}=9$; $6 \mathrm{~Gy}, \mathrm{n}=9$ ) were analysed. As shown in Fig. 4a, the concentration of Flt3lg was $275 \pm 79 \mathrm{pg} / \mathrm{ml}$ (range, $\sim 160$ to $\sim 410$ ) in the sham group, increased to $687 \pm 166 \mathrm{pg} / \mathrm{ml}$ (range, $\sim 530$ to $\sim 1,050$ ) in the 2 Gy group, and further increased to $1442 \pm 410 \mathrm{pg} / \mathrm{ml}$ (range, $\sim 800$ to $\sim 2,000$ ) in the 6 Gy group, with significant concentration increases for each radiation dose group $(\mathrm{p}<0.0001)$. The concentration of Saal (Fig. 4b) was $3.5 \pm 3.8 \mu \mathrm{g} / \mathrm{ml}$ (range, $\sim 0.3$

b

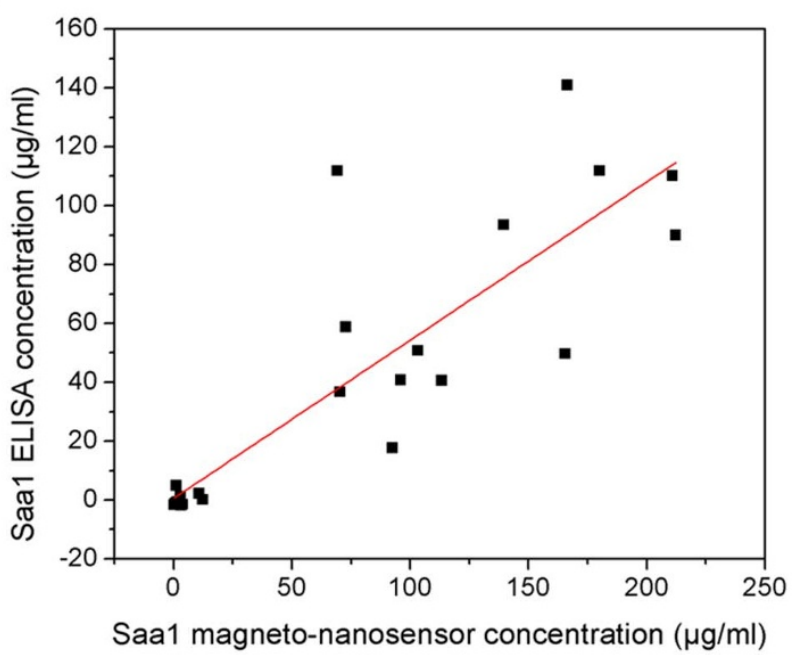

Figure 3 Correlation scatter plots between ELISA and magneto-nanosensor biochip assay for mouse (a) Flt3lg and (b) Saa1. 
a

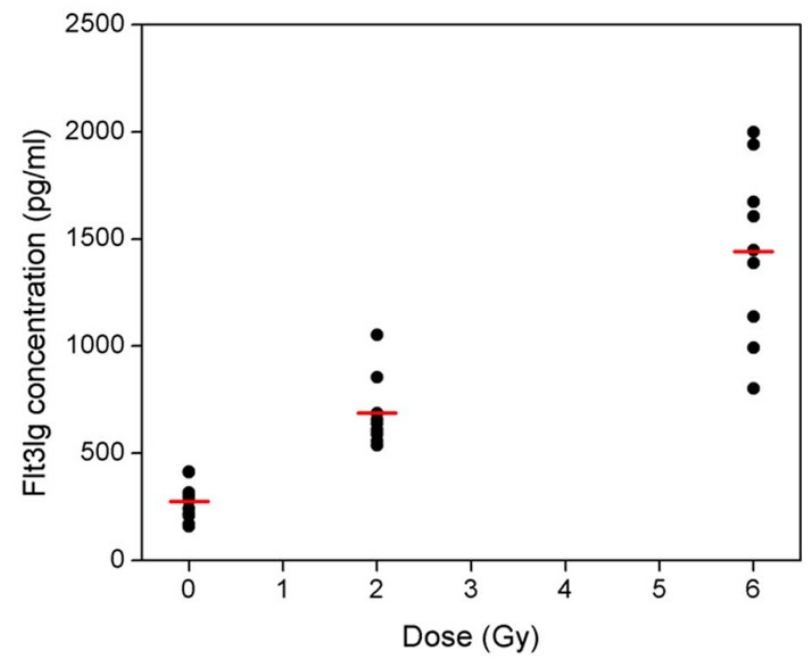

b

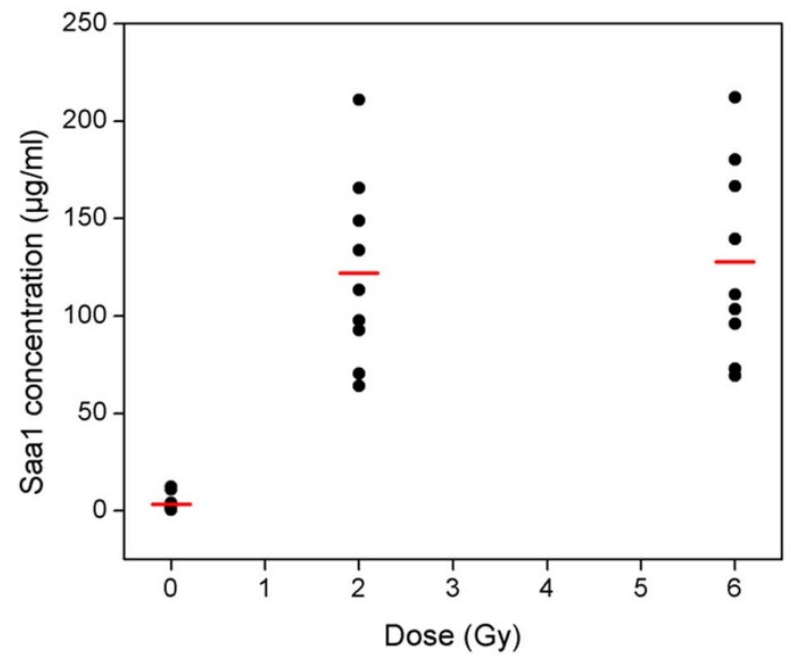

Figure $4 \mid$ Radiation dose response behaviour of mouse (a) Flt3lg and (b) Saa1 measured using magneto-nanosensor biochips for 0, 2, and 6 Gy irradiated C57BL/6J mice at 24 hours post-irradiation. Red bars indicate average values, and each black dot represents one animal.

to $\sim 13$ ) in the same sham samples, and increased about 30 fold after 2 Gy irradiation (average, $122 \pm 48 \mu \mathrm{g} / \mathrm{ml}$; range, $\sim 60$ to $\sim 210$ ), but with no further increase at $6 \mathrm{~Gy}$ (average, $128 \pm 50 \mu \mathrm{g} / \mathrm{ml}$; range, $\sim 70$ to $\sim 210$ ). For Saa1, the sham-irradiated and irradiated ( 2 or 6 Gy) groups could be fully distinguished since their Saal concentration ranges did not overlap. However, the Saal concentration difference between 2 and 6 Gy groups was not significant $(p=0.8)$.
Dose response change with time after exposure. We then investigated the changes in dose response of Flt3lg and Saal during the first week after TBI exposure using repeated venous blood draws from living mice. For this experiment, $\sim 25 \mathrm{ul}$ of blood was obtained from the saphenous vein of each mouse at various time points after irradiation, and plasma was isolated by centrifugation. A total of 210 plasma samples were collected from 35 female mice at 6 times after exposure ( 8 days prior to exposure and a
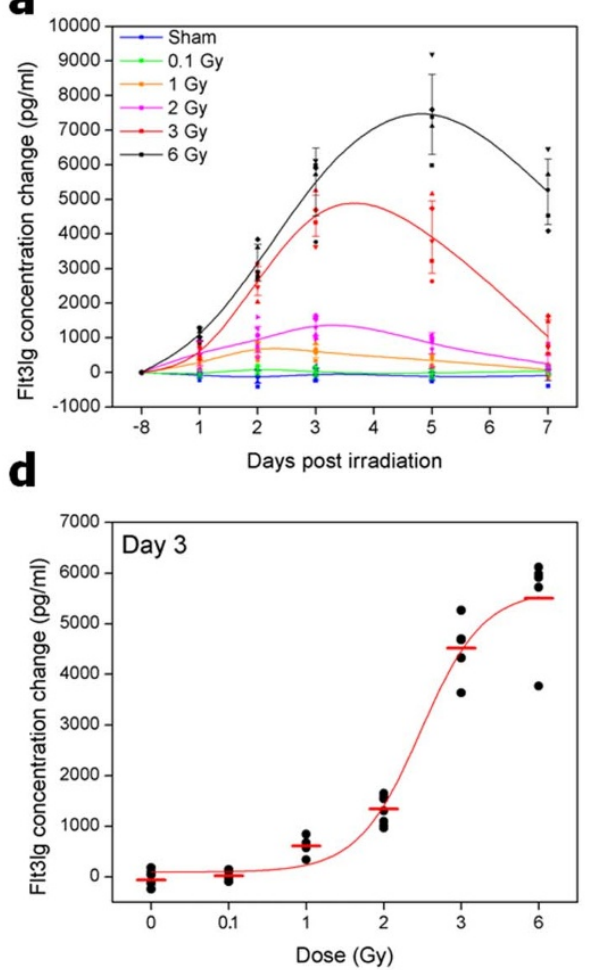

b
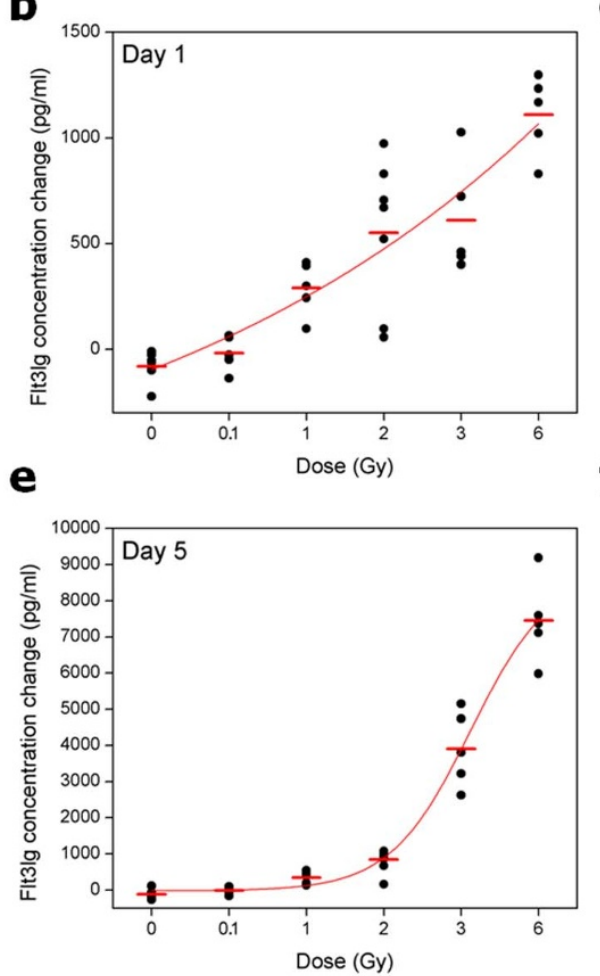

C
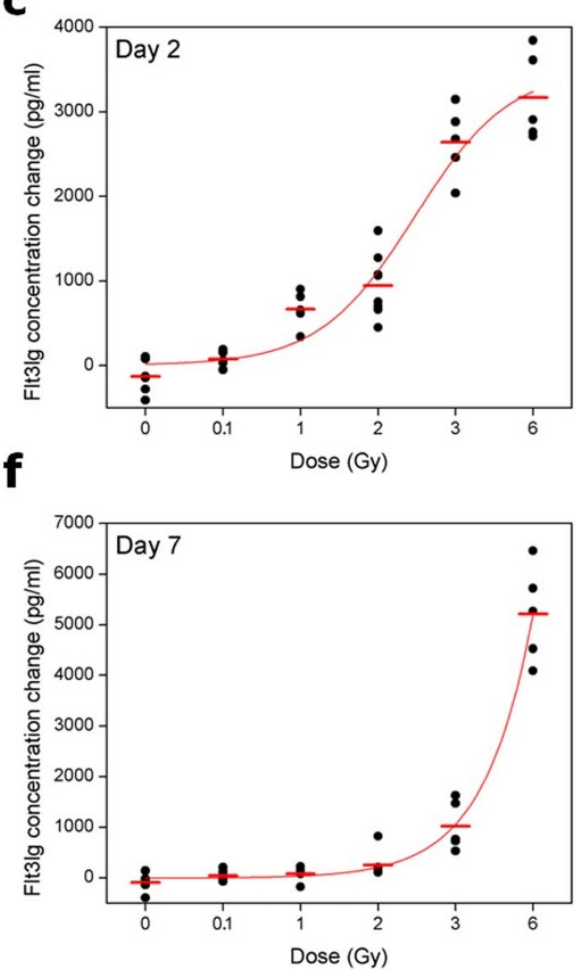

Figure 5 | (a) Radiation time response behaviour of mouse Flt3lg measured using magneto-nanosensor biochips for sham, 0.1, 1, 2, 3, and 6 Gy (blue, green, orange, magenta, red, and black, respectively) irradiated C57BL/6J mice at 8 days before, 1, 2, 3, 5, and 7 days post-irradiation. Error bars indicate \pm 1 standard deviation for each radiation exposure group. Dose response behaviours of mouse Flt3lg at days (b) 1, (c) 2, (d) 3, (e) 5, and (f) 7 after exposure to radiation. Red bars indicate average values, and each black dot represents one animal. Red lines are dose response fitting curves. 
at $1,2,3,5$, and 7 days after exposure) at doses from 10 cGy to $6 \mathrm{~Gy}$ (sham, $\mathrm{n}=7 ; 10$ cGy and $1 \mathrm{~Gy}, \mathrm{n}=5 ; 2 \mathrm{~Gy}, \mathrm{n}=8 ; 3$ and $6 \mathrm{~Gy}, \mathrm{n}=$ 5). All measurements were blinded so that the experimenter measured magnetic signals without knowing the actual radiation doses.

Fig. 5 shows the time response for Flt3lg. As can be seen in Fig. 5a, the Flt $3 \lg$ concentration remained at similar values as the basal level for sham-irradiated samples, but increased to $\sim 750,1420,4520$, and $7450 \mathrm{pg} / \mathrm{ml}$ as maximum average changes for 1, 2, 3, and 6 Gy irradiated samples, respectively. Fig. 5b, c, d, e, and f show the Flt3lg dose responses at days 1, 2, 3, 5, and 7 after exposure, respectively, and $\mathrm{P}$ values associated with two-tailed Student's t-test are presented in Supplementary Table S1 and S2. Exposure groups irradiated at higher doses showed higher Flt3lg concentrations at all time points, and significant concentration differences against sham at $\geq 1$ Gy in the time window of 1 to 7 days after exposure other than 1 Gy at day 7 could be seen (Supplementary Table S1, p $<0.0001$, except 1 Gy at day 1 and 5 where $p=0.0070$ and 0.0006 , respectively). However, the Flt3lg concentration change was not significant for $10 \mathrm{cGy}$ exposure group. $(\mathrm{p}=0.1)$. Supplementary Table S2 lists the $\mathrm{p}$ values for comparison of immediately lower doses on the same day. Significant concentration differences $(\mathrm{p}<0.05)$ still could be observed except between 2 and 3 Gy samples at day $1(\mathrm{p}=0.4)$, and between 3 and 6 Gy samples at day 2 and $3(\mathrm{p}=0.2$ and 0.1 , respectively).

Supplementary Table S3 lists the sensitivity, specificity, positive predictive values (PPV), and negative predictive values (NPV) obtained by dose discriminant analysis using Flt3lg for sham, 1, 2, 3 , and 6 Gy irradiated samples at day 1, 2, 3, 5, and 7 after irradiation. 0.1 Gy samples were excluded from the dose discriminant analysis because their Flt3lg concentration changes from sham samples were insignificant. Here, the DISCRIM procedure in SAS software was used to define a discriminant function, which subsequently calculates the probability of measured concentrations at a given time point being classified into each radiation dose group. Many of the dose groups could be classified with high sensitivity $(0.8 \sim 1)$, specificity $(0.8 \sim 1)$, PPV $(0.7 \sim 1)$, and NPV $(0.7 \sim 1)$, showing excellent dose discrimination among the dose groups. There were some radiation dose groups showing sensitivity and PPV lower than 0.5 (2 Gy at day 1, 6 Gy at day 2, and sham and 1 Gy at day 7), which were caused by similar Flt3lg concentrations between these radiation dose groups and other groups at the same day post irradiation, as can be seen in Figs. $5 \mathrm{~b}, \mathrm{c}$, and $\mathrm{f}$, respectively.

Fig. 6 shows the time response for Saa1. As can be seen in Fig. 6a, a sharp increase in plasma concentration of $\sim 80 \mu \mathrm{g} / \mathrm{ml}$ on average was observed for 2, 3, and 6 Gy irradiated samples at day 1 after irradiation, after which the concentration decreased to near basal level by 3 days after irradiation. Fig. $6 \mathrm{~b}$ and $\mathrm{c}$ show the Saal dose responses at days 1 and 2 after exposure, respectively. Saal concentrations at day 2 for doses $\geq 1$ Gy still remained higher than sham levels even though they were not as high as those at day 1 . Supplementary Table S4 presents the p values associated with twotailed Student's t-test. Significant concentration differences between sham and $\geq 1$ Gy dose groups could be seen for the first two days after exposure $(\mathrm{p}<0.0001)$, but the concentration change was not significant for $10 \mathrm{cGy}$ group ( $\mathrm{p}=0.4067$ at day 1$)$. Supplementary Table S5 lists the p values for comparison of immediately lower doses on the same day.

Supplementary Table S6 lists the sensitivity, specificity, PPV, and NPV obtained by dose discriminant analysis using Saal for sham, 1 , 2, 3, and 6 Gy irradiated samples at day 1 and 2 after irradiation. Although the dose discrimination power of Saal concentrations for distinguishing each radiation dose groups was not as good as that of Flt3lg concentrations, Saal concentrations still could be used to discriminate sham-irradiated samples from irradiated ( $\geq 1$ Gy) samples with high sensitivity $(=1)$, specificity $(0.87 \sim 1)$, $\operatorname{PPV}(0.7 \sim 1)$, and NPV $(=1)$. Supplementary Tables S7 and S8 list the measurement data presented in Figs. 5 and 6.

\section{Discussion}

We developed a magneto-nanosensor platform based on the GMR effect to measure concentrations of multiple radiation-responsive proteins in peripheral blood and then demonstrated the robustness of the biochip platform by measuring two radiation biomarkers using small volume blood samples collected from the in vivo mouse radiation model.

Our magneto-nanosensor biochip has a size of $10 \mathrm{~mm} \times 12 \mathrm{~mm}$ and has an array of 64 individually addressable sensors (each sensor is made of 48 spin-valve strips with a size of $90 \mu \mathrm{m} \times 0.75 \mu \mathrm{m})$ covered by ultrathin and biochemically stable silicon oxide passivation $^{22}$. In the experiments, some sensors were additionally covered by epoxy or coated with BSA or biotin-BSA. Epoxy covered sensors served as electrical reference sensors. Electrical background noise was removed by subtracting the average signal of the epoxy-covered sensors from all the other signals. Bovine serum albumin (BSA)coated sensors were used as negative control sensors, since they were blocked and thus prevented from specifically capturing either target antigen or streptavidin-tagged magnetic nanoparticles. The very small signals that appeared on BSA-coated sensors clearly indicated that the observed signals from the capture antibody-coated sensors were not a result of non-specific binding of the analyte or magnetic nanoparticles. Biotin-coated sensors were used as positive a

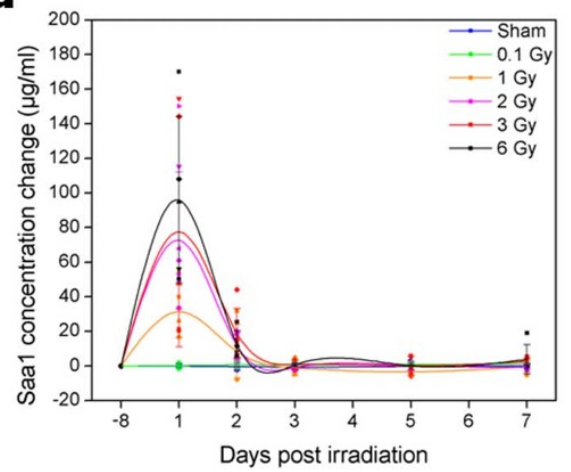

b

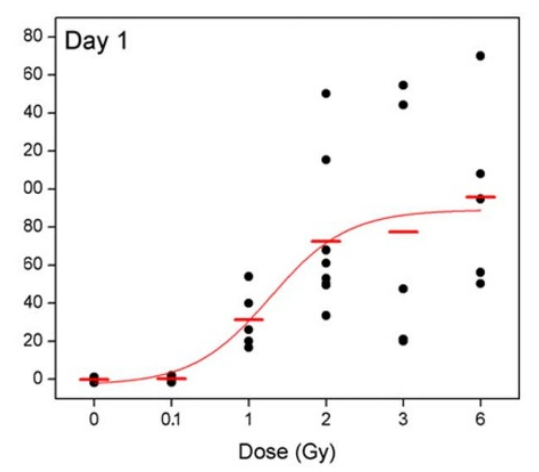

\section{C}

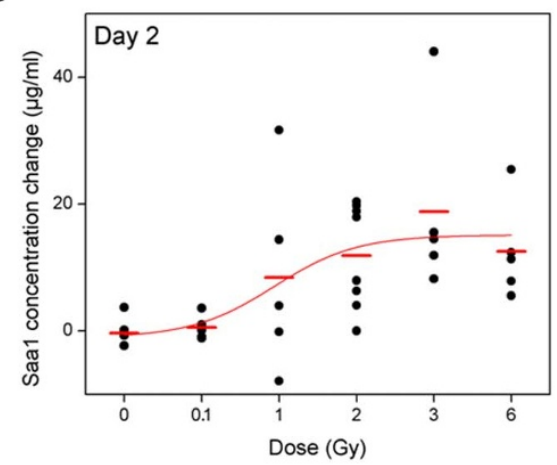

Figure 6 (a) Radiation time response behaviour of mouse Saal measured using magneto-nanosensor biochips for sham, 0.1, 1, 2, 3, and 6 Gy

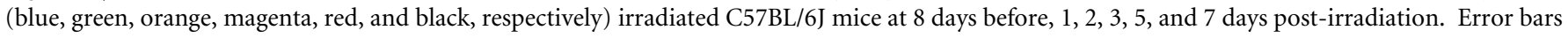
indicate \pm 1 standard deviation for each radiation exposure group. Dose response behaviours of mouse Saal at days (b) 1 and (c) 2 after exposure to radiation. Red bars indicate average values, and each black dot represents one animal. Red lines are dose response fitting curves. 
functional controls because of their ability to specifically capture streptavidin-tagged magnetic nanoparticles, and they showed very large signals as expected.

There were some discrepancies between the concentrations measured on magneto-nanosensor chips and ELISA, which might result from differences in antibody surface conjugation or from differences in the signal generation mechanism. It has been proposed that different surface modification methods or assay techniques could result in a different quantification of analyte ${ }^{39-42}$. However, the calculated values of Spearman's rank correlation coefficient $(\rho=0.915$ for Flt3lg and $\rho=0.845$ for Saa1) clearly show that the concentrations determined using magneto-nanosensor biochip and ELISA have significantly positive correlation. Although we were not able to perform ELISA measurements in parallel with the magneto-nanosensor biochip measurements for the in vivo irradiation time response mouse model samples due to limited blood volume permitted by IACUC (Institutional Animal Care and Use Committee), the significant correlation between ELISA and the magneto-nanosensor shown in Fig. 3 ensures the validity of the magneto-nanosensor for measuring plasma samples collected from radiation exposure studies.

We measured the dose and time dependent concentration changes of plasma Flt3lg and Saal in C57BL/6J strain mice during the first week after radiation exposure. Flt3lg showed elevated levels at all time points in the time windows of 1 to 7 days after exposure to radiation doses $\geq 1 \mathrm{~Gy}$, and their maximum changes increased with increasing radiation doses. Previous studies also reported the increase of Flt3lg level after exposure to different radiation doses, although the maximum and temporal concentration changes varied among different mice strains $s^{31,43}$. Saal showed elevated levels only during the first two days after exposure exhibiting maximum changes at day 1 . Dose dependent increase of serum amyloid A at day 1 was reported in a study using BALB/c strain mice, although temporal changes were not measured ${ }^{44}$.

In the dose response study, although the general trend of Flt3lg concentration change was elevation of Flt3lg concentration with increasing irradiation dose, there was some overlap in the concentration ranges of 2 and 6 Gy dose groups. Saal concentration also increased after irradiation, but Saal concentration ranges of 2 and 6 Gy dose groups were very similar. These different levels of Flt3lg and Saal upregulation after irradiation suggest inter-individual variations in radiation response $\mathrm{e}^{45-47}$. It has been known that genetically diverse individuals respond differently to radiation exposure, revealing less than $\sim 10 \%$ of individuals are hyper-radiosensitive, $\sim 70 \%$ are normally sensitive, and the remaining $20 \%$ are considered radioresistant ${ }^{26,47}$. However, because the mice used in this study were genetically identical, the observed variations seem to be caused by non-genetic fluctuations.

In the time response study, it is worth noting the relatively large concentration difference between the ranges of 2 and 3 Gy irradiated samples, implying relatively much higher damage is expected when irradiated by doses $\geq 3$ Gy. Since Flt3lg and Saal showed different level changes over time after irradiation in our study (Flt3lg level increases up to $3 \sim 5$ days before starting to decrease whereas elevated Saal level persists for only two days), it is possible to use both biomarkers together for determining the radiation exposure time point retrospectively. For example, 2 Gy irradiated samples at day 1 and 7 after irradiation might be undistinguishable using only Flt3lg because of their similar Flt3lg concentrations at day 1 and 7 . However, they would be distinguishable if we measure Saal along with Flt3lg since day 1 samples would have higher Saa1 levels than those in the day 7 samples. In general, the use of multiple biomarkers enables higher accuracy for diagnostic tests than do single biomarkers $^{6,26,27,48}$. For samples exposed to 1 or 2 Gy, considering both Flt3lg and Saal can improve the classification, especially on day 1 and 2, due to their different time dependent concentration changes as mentioned above (Supplementary Table S9). Discrimination error rates from day 5 were higher than earlier days due to similar Saal concentrations and decreasing Flt3lg concentration differences among samples of different doses. For samples exposed to $\geq 3 \mathrm{~Gy}$, on the other hand, adding Saal as a secondary biomarker did not improve the classification as Flt3lg had a discrimination power much higher than that of Saa1 (Supplementary Table S10).

In summary, we have developed a novel protein immunoassay platform using magneto-nanosensor biochips, and have demonstrated its utility by measuring Flt3lg and Saal biomarkers in an in vivo mouse model. For the same capture and detection antibodies, the ELISA and magneto-nanosensor results were highly correlated, and the magneto-nanosensor showed a large dynamic range $\mathrm{e}^{20,22}$. We also monitored dose dependent and temporal upregulation of Flt3lg and Saal in radiation dose and time response studies. Flt3lg concentration change showed excellent dose discrimination over a week after radiation exposure, and Saal dose response was apparent for the first two days after exposure. A multiplex assay with both proteins could improve classification accuracy of radiation exposure. Since our platform is applicable for all protein biomarkers, we believe that the magneto-nanosensor biochips hold great potentials for medical diagnosis and clinical research, including but not limited to radiation biomarker validation and early radiation triage.

\section{Methods}

Materials. Anti-mouse Flt3lg antibody, biotinylated anti-mouse Flt3lg antibody, mouse Flt3lg standard (all from R\&D systems, DY427), anti-mouse Saal antibody (R\&D systems, AF2948), biotinylated anti-mouse Saal antibody (R\&D systems, BAF2948), mouse Saa1 standard (ALPCO diagnostics, 41-SAAMS-E01), poly(allylamine hydrochloride) (Polyscience), poly(ethylene-alt-maleic anhydride) (Aldrich), $1 \times$ phosphate buffered saline (PBS) (Invitrogen), 1-ethyl-3-(3dimethylaminopropyl)carbodiimide hydrochloride (EDC) (Thermo scientific), Nhydroxysuccinimide (NHS) (Aldrich), 1\% bovine serum albumin (BSA) (Aldrich), biotinylated bovine serum albumin (biotin-BSA) (Pierce), Tween 20 (Aldrich), and streptavidin-coated MicroBeads (Miltenyi, 130-048-101) were used as received, and without further purification. Mouse (C57BL/6J males and females) plasma samples were prepared by depositing blood, with heparin added as an anticoagulant, into Microvette tubes (Sarstedt) and centrifuging.

Mouse radiation model for collection of blood plasma. The use of animals in this study was approved by the Institutional Animal Care and Use Committee at both Lawrence Berkeley National Laboratory and Stanford University.

Dose response study. Male and female C57BL/6J mice between 8 and 10 weeks of age were irradiated and blood sampled similar to that previously described ${ }^{26,27}$. Briefly mice were exposed to sham, 2, or 6 Gy irradiation using 320-kVp X-ray (Pantak) at dose rates of $0.75 \mathrm{~Gy} / \mathrm{min}$ for $2 \mathrm{~Gy}$ and $1.9 \mathrm{~Gy} / \mathrm{min}$ for $6 \mathrm{~Gy}$. The consistency of the delivered dose was monitored using a dosimeter in every $2 \sim 3$ radiations. Blood was collected from euthanized mice by cardiac puncture $24 \mathrm{hr}$ after irradiation using heparin-wetted syringes and $25 \mathrm{G}$ needles and transferred into Eppendorf tubes containing $10 \mu \mathrm{l}$ of heparin. Blood was then transferred to another centrifuge tube and layered on top of $1 \mathrm{ml}$ HISTOPAQUE 1083 (Sigma). Plasma was isolated by centrifugation and stored at $-80^{\circ} \mathrm{C}$ prior to analysis.

Post-exposure time response study. C57BL/6J male and female mice were irradiated with $0,0.1,1,2,3$, or $6 \mathrm{~Gy} \mathrm{X}$-ray of $320-\mathrm{kVp}$ at a dose rate of $0.75 \mathrm{~Gy} / \mathrm{min}$ except the $6 \mathrm{~Gy}$ exposure, which was performed at a dose rate of $1.9 \mathrm{~Gy} / \mathrm{min}$. About $25 \mu \mathrm{l}$ (range, $\sim 13$ to $\sim 40$ ) of blood were collected from the saphenous vein $\left(\right.$ Hem et al. ${ }^{49}$ ) at $1,2,3,5$, and 7 days after irradiation using an heparinised eppendorf pipette tip and transferred into Starstedt Microvette CB 300 capillary tubes, and plasma isolated after centrifugation and stored at $-80^{\circ} \mathrm{C}$ prior to analysis. Pre-irradiation samples were collected from all mice 8 days before irradiation or sham-irradiation to establish baseline levels of Flt3lg and Saa1 for each mouse.

Magneto-nanosensor chip surface preparation. The magneto-nanosensor chip was fabricated by previously reported method ${ }^{22}$. The sensor chip surface was washed with acetone, methanol, and isopropanol. Subsequently, the surface was further cleaned by exposing to oxygen plasma (Harrick Plasma, PDC-32G) for 3 minutes. Then, the surface was immersed in a $1 \%$ aqueous solution of poly(allylamine hydrochloride) for 5 minutes, followed by rinsing with deionised water. The sensor chip was baked at $120^{\circ} \mathrm{C}$ for 1 hour. After incubation in a $2 \%$ aqueous solution of poly(ethylene-altmaleic anhydride), the surface was washed again with deionised water and activated by adding a mixture of 1-ethyl-3-[3-dimethylaminopropyl]carbodiimide hydrochloride and $\mathrm{N}$-hydroxysuccinimide in deionised water. A robotic spotter (Scienion, sciFlexarrayer) was then used to deposit capture antibody solution on the sensor chip surface. A PBS solution of anti-mouse Flt3lg $(0.5 \mathrm{mg} / \mathrm{ml})$ and a PBS solution of anti-mouse Saal $(1 \mathrm{mg} / \mathrm{ml})$ were deposited on at least 8 sensors. Also, 
$0.1 \%$ PBS solutions of BSA and biotin-BSA were placed over 6 sensors as negative and positive controls, respectively. Reference sensors were covered with epoxy to measure electrical background signals. Finally, the sensor chip was stored in a humidity chamber at $4^{\circ} \mathrm{C}$ before use.

Flt3lg/Saal duplex-assay protocol. After washing the sensor chip surface with a washing buffer $(0.1 \% \mathrm{BSA}$ and $0.05 \%$ Tween 20 in PBS), the surface was blocked with $1 \%$ BSA for 1 hour. Then, the surface was washed again and immersed in a $4000 \times$ diluted sample (diluted in dilution buffer, $0.1 \%$ BSA and $0.05 \%$ Tween 20 in PBS) for 2 hours. The sample solution was washed away with the washing buffer and a Saal detection antibody solution with a concentration of $5 \mu \mathrm{g} / \mathrm{ml}$ was added. Following 1 hour incubation with the detection antibody, the surface was washed again before measuring Saal signal from the chip. After completing the real-time signal collection for Saal, similar procedures excluding the blocking step were used with a $5 \times$ diluted sample and an Flt3lg detection antibody solution before collecting Flt3lg signals.

Analyte quantification. The electrical read-out system for the magneto-nanosensor chip was implemented according to Hall et al. ${ }^{37,38}$. Streptavidin-coated magnetic nanoparticles (Miltenyi, streptavidin MicroBeads) were added to sensor chip to induce an analyte concentration-dependent signal change. The measured signals were converted to corresponding concentrations using the standard curves for each biomarker.

ELISA measurements. Plasma protein biomarkers (i.e., Flt3lg and Saa1) were measured using ELISA kits (Flt3lg: R\&D Systems, DY427; Saa1: ALPCO, 41-SAAMSE01) according to the manufacturer's instructions. Three replicate measurements were determined for each sample and standards. The Flt3lg and Saal concentrations in plasma samples were determined via the generated calibration curve for standard proteins.

Statistical data analysis. Statistical software MedCalc was used for statistical analysis. Two-sided Student's $t$-test was used to determine significant difference between two groups. $P$ values of $<0.05$ were considered statistically significant. Correlation analysis between magneto-nanosensor and ELISA was done using non-parametric Spearman rank correlation, since it did not require normality assumption and the number of samples was relatively small. Statistical software SAS was used for the DISCRIM procedure to classify the radiation exposure time points under a given radiation dose.

1. Brenner, D. J. et al. Cancer risks attributable to low doses of ionizing radiation assessing what we really know. Proc. Natl. Acad. Sci. USA 100, 13761-13766 (2003).

2. Atkinson, J. et al. A mitochondria-targeted inhibitor of cytochrome $c$ peroxidase mitigates radiation-induced death. Nat. Commun. 2, 497 (2011).

3. Blakely, W. F., Salter, C. A. \& Prasanna, P. G. S. Early-response biological dosimetry- recommended countermeasure enhancements for mass-casualty radiological incidents and terrorism. Health Phys. 89, 494-504 (2004).

4. Pinto, M. M. P. de L., Santos, N. F. G. \& Amaral, A. Current status of biodosimetry based on standard cytogenetic methods. Radiat. Environ. Biophys. 49, 567-581 (2010).

5. Fenech, M. Current status, new frontiers and challenges in radiation biodosimetry using cytogenic, transcriptomic and proteomic technologies. Radiat. Meas. 46, 737-741 (2011).

6. Budworth, H. et al. DNA repair and cell cycle biomarkers of radiation exposure and inflammation stress in human blood. PLoS One 7, e48619 (2012).

7. Rifai, N., Gillette, M. A. \& Carr, S. A. Protein biomarker discovery and validation: the long and uncertain path to clinical utility. Nat. Biotechnol. 24, 971-983 (2006).

8. Li, J. et al. Identification of high-quality cancer prognostic markers and metastasis network modules. Nat. Commun. 1, 34 (2010).

9. Dalton, W. S. \& Friend, S. H. Cancer biomarkers-an invitation to the table. Science 312, 1165-1168 (2006).

10. Gosling, J. P. A decade of development in immunoassay methodology. Clin. Chem. 36, 1408-1427 (1990)

11. Rissin, D. M. et al. Single-molecule enzyme-linked immunosorbent assay detects serum proteins at subfemtomolar concentrations. Nat. Biotechnol. 28, 595-600 (2010)

12. Kingsmore, S. F. Multiplexed protein measurement: technologies and applications of protein and antibody arrays. Nat. Rev. Drug. Discov. 5, 310-321 (2010).

13. Tabakman, S. M. et al. Plasmonic substrates for multiplexed protein microarrays with femtomolar sensitivity and broad dynamic range. Nat. Commun. 2, 466 (2011)

14. Cui, Y., Wei, Q., Park, H. \& Lieber, C. M. Nanowire nanosensors for highly sensitive and selective detection of biological and chemical species. Science 293, 1289-1292 (2001).

15. Backmann, N. et al. A label-free immunosensor array using single-chain antibody fragments. Proc. Natl. Acad. Sci. USA 102, 14587-14592 (2005).

16. Stern, E. et al. Label-free biomarker detection from whole blood. Nat. Nanotechnol. 5, 138-142 (2010).

17. Cai, D. et al. A molecular-imprint nanosensor for ultrasensitive detection of proteins. Nat. Nanotechnol. 5, 597-601 (2010).
18. Fan, C., Plaxco, K. W. \& Heeger, A. J. Electrochemical interrogation of conformational changes as a reagentless method for the sequence-specific detection of DNA. Proc. Natl. Acad. Sci. USA 100, 9134-9137 (2003).

19. Drummond, T. G., Hill, M. G. \& Barton, J. K. Electrochemical DNA sensors. Nat. Biotechnol. 21, 1192-1199 (2003).

20. Gaster, R. S. et al. Matrix-insensitive protein assays push the limits of biosensors in medicine. Nat. Med. 15, 1327-1332 (2009).

21. Marchetti, F., Coleman, M. A., Jones, I. M. \& Wyrobek, A. J. Candidate protein biodosimeters of human exposure to ionizing radiation. Int. J. Radiat. Biol. 82, 605-639 (2006).

22. Osterfeld, S. J. et al. Multiplex protein assays based on real-time magnetic nanotag sensing. Proc. Natl. Acad. Sci. USA 105, 20637-20640 (2008).

23. Gaster, R. S., Hall, D. A. \& Wang, S. X. Autoassembly protein arrays for analyzing antibody cross-reactivity. Nano. Lett. 11, 2579-2583 (2011).

24. Gaster, R. S. et al. Quantification of protein interactions and solution transport using high-density GMR sensor arrays. Nat. Nanotechnol. 6, 314-320 (2011).

25. Gaster, R. S., Hall, D. A. \& Wang, S. X. nanoLAB: an ultraportable, handheld diagnostic laboratory for global health. Lab. Chip. 11, 950-956 (2011).

26. Ossetrova, N. I. \& Blakely, W. F. Multiple blood-proteins approach for earlyresponse exposure assessment using an in vivo murine radiation model. Int. J. Radiat. Biol. 85, 83-850 (2009).

27. Blakely, W. F., Sandgren, D. J., Nagy, V., Kim, S.-Y. \& Ossetrova, N. I. Murine partial-body radiation exposure model for biodosimetry studies - preliminary report. Radiat. Meas. 46, 898-902 (2011).

28. Lee, H.-J. et al. Identification of possible candidate biomarkers for local or whole body radiation exposure in C57BL/6 mice. Int. J. Radiat. Oncol. Biol. Phys. 69, 1272-1281 (2007).

29. Agrawal, D. K., Hopfenspirger, M. T., Chavez, J. \& Talmadge, J. E. Flt3 ligand: a novel cytokine prevents allergic asthma in a mouse model. Int. Immunopharmacol. 1, 2081-2089 (2001).

30. Hannum, C. et al. Ligand for FLT3/FLK2 receptor tyrosine kinase regulates growth of haematopoietic stem cells and is encoded by variant RNAs. Nature 368, 643-648 (1994)

31. Prat, M. et al. Radiation-induced increase in plasma Flt3 ligand concentration in mice: evidence for the implication of several cell types. Radiat. Res. 163, 408-417 (2005).

32. Gratwohl, A. et al. FLT-3 ligand provides hematopoietic protection from total body irradiation in rabbits. Blood 92, 765-769 (1998).

33. Xu, Y., Yamada, T., Satoh, T. \& Okuda, Y. Measurement of serum amyloid Al (SAA1), a major isotype of acute phase SAA. Clin. Chem. Lab. Med. 44, 59-63 (2006).

34. Sung, H.-J. et al. Identification and validation of SAA as a potential lung cancer biomarker and its involvement in metastatic pathogenesis of lung cancer J. Proteome. Res. 10, 1383-1395 (2011).

35. Kluve-Beckerman, B., Yamada, T., Hardwick, J., Liepnieks, J. J. \& Benson, M. D. Differential plasma clearance of murine acute-phase serum amyloid A proteins SAA1 and SAA2. Biochem. J. 322, 663-669 (1997).

36. Goltry, K. L., Epperly, M. W. \& Greenberger, J. S. Induction of serum amyloid A inflammatory response genes in irradiated bone marrow cells. Radiat. Res. 149, 570-578 (1998).

37. Hall, D. A. et al. GMR biosensor arrays: a system perspective. Biosens. Bioelectron. 25, 2051-2057 (2010)

38. Hall, D. A. et al. GMR biosensor arrays: correction techniques for reproducibility and enhanced sensitivity. Biosens. Bioelectron. 25, 2177-2181 (2010).

39. Jonkheijm, P., Weinrich, D., Schröder, H., Niemeyer, C. M. \& Waldmann, H. Chemical strategies for generating protein biochips. Angew. Chem. Int. Ed. Engl. 47, 9618-9647 (2008)

40. Goddard, J. M. \& Erickson, D. Bioconjugation techniques for microfluidic biosensors. Anal. Bioanal. Chem. 394, 469-479 (2009).

41. Malan, A. K., Martins, T. B., Jaskowski, T. D., Hill, H. R. \& Litwin, C. M. Comparison of two commercial enzyme-linked immunosorbent assays with an immunofluorescence assay for detection of legionella pneumophila types 1 to 6 . J. Clin. Microbiol. 41, 3060-3063 (2003).

42. Gilbert, N. et al. Comparison of commercial assays for the quantification of HBV DNA load in health care workers: calibration differences. J. Virol. Methods 100 37-47 (2002)

43. Sproull, M., Avondoglio, D., Meushaw, T., Shankavaram, U. \& Camphausen, K Plasma Flt3-ligand as a biomarker for radiation exposure. Int. J. Radiat. Oncol. Biol. Phys. 78, S635 (2010).

44. Ossetrova, N. I., Sandgren, D. J., Gallego, S. \& Blakely, W. F. Combined approach of hematological biomarkers and plasma protein SAA for improvement of radiation dose assessment triage in biodosimetry applications. Health Phys. 98, 204-208 (2010).

45. Kato, T. A. et al. Variations in radiosensitivity among individuals: a potential impact on risk assessment? Health Phys. 97, 470-480 (2009).

46. Tucker, S. L., Turesson, I. \& Thames, H. D. Evidence for individual differences in the radiosensitivity of human skin. Eur. J. Cancer 11, 1783-1791 (1992).

47. Schnarr, K., Dayes, I., Sathya, J. \& Boreham. D. Individual radiosensitivity and its relevance to health physics. Dose-Response 5, 333-348 (2007).

48. Ossetrova, N. I., Farese, A. M., MacVittie, T. J., Manglapus, G. L. \& Blakely, W. F. The use of discriminant analysis for evaluation of early-response multiple 
biomarkers of radiation exposure using non-human primate 6-Gy whole-body radiation model. Radiat. Meas. 42, 1158-1163 (2007).

49. Hem, A., Smith, A. J. \& Solber, P. Saphenous vein puncture for blood sampling of the mouse, rat, hamster, gerbil, guinea pig, ferret and mink. Lab. Animals. 32, 364-368 (1998).

\section{Acknowledgments}

This project has been funded in whole or in part by Federal funds from the Biomedical Advanced Research and Development Authority, Office of the Assistant Secretary for Preparedness and Response, Office of the Secretary, Department of Health and Human Services, under Contract No. HHSO100201000006C. This work was performed under the auspices of the U.S. Department of Energy by the University of California, Lawrence Berkeley National Laboratory under DOE contract No. DE-AC02-05CH11231 and with funding support from BAA-BARDA-09-36. The Armed Forces Radiobiology Research Institute, under work units RAB4AP and RBB4AR, provided the support for one of the co-authors involved in this research (W.B.). The views expressed here are those of the authors; no endorsement by the U.S. Department of Defense or the U.S. government has been given or inferred. The work at Stanford also benefited from the National Cancer Institute grants Physical Science Oncology Center (U54CA143907), Center for Cancer Nanotechnology Excellence (U54CA151459), Innovative Molecular Analysis Technologies (R33CA138330).

\section{Author contributions}

D.K., F.M., W.F.B., A.J.W. and S.X.W. designed the research. D.K., F.M., Z.C., S.Z., S.B., B.M., S.T., K.R. and M.C. performed the experiments. D.K., F.M., R.J.W., D.A.H., R.S.G., J.L., J.W., S.J.O., H.Y. and A.J.W. contributed new reagents/analytic tools. D.K., F.M., R.J.W., R.M.W., W.F.B., L.P., A.M.S., A.J.W. and S.X.W. analysed the data. D.K., F.M. and S.X.W. wrote the initial draft of the paper and all authors commented on the paper.

\section{Additional information}

Supplementary information accompanies this paper at http://www.nature.com/ scientificreports

Competing financial interests: The authors declare no competing financial interests. How to cite this article: Kim, D. et al. Nanosensor dosimetry of mouse blood proteins after exposure to ionizing radiation. Sci. Rep. 3, 2234; DOI:10.1038/srep02234 (2013).

sa NonCommercial-ShareAlike 3.0 Unported license. To view a copy of this license, visit http://creativecommons.org/licenses/by-nc-sa/3.0 